\title{
What the ancients believed
}

COSMOLOGY is historically an episodic field. Over the long period going back to before ancient Greece, the current view of how the Universe is constructed has been drastically changed by the arrival of new techniques and the accumulation of data. Much has changed since some among the ancients likened the stars in the sky to lanterns hung from a spherical and moving ceiling that is itself invisible.

But by the time of Aristarchus $(310-230 \mathrm{BC})$, the Greeks had a clear picture of a heliocentric Solar System, with the distance of the Sun estimated at between 18 and 20 times that of the Moon. Archimedes, in a manuscript of $215 \mathrm{BC}$, gives an interpretation of Aristarchus in which the Sun is at the centre of a static spherical Universe, with the Earth and the other planets in orbits about it. The fixed stars are fixed.

Erastosthenes, one of the first directors of the great library at Alexandria, provided this cosmology with a yardstick by a measurement of the diameter of the Earth, afterwards much improved by observations by Hipparchus of eclipses, notably the solar eclipse of 129 BC. Hipparchus seems also to have begun a catalogue of the fixed stars, later used by Ptolemy (after the Romans had taken over at Alexandria) in his more extensive compilation of the positions of more than 1,000 stars.

It is far from clear why Ptolemy, apparently ${ }^{1}$ following the lead of Hipparchus, had retreated by the second century AD to a view of the world that restored the Earth to centre-stage (and described the motion of the planets by his notoriously complicated system of epicycles), but that was the position of Christendom when, much later, Copernicus reintroduced heliocentric cosmology.

Copernicus's book was published in the year of his death in 1543. The heroic century and a half that followed included Galileo's demonstration of his version of the Equivalence Principle (that gravitational and inertial mass are identical) and culminated with Newton's theory of gravitation in 1687 (published, together with the laws of motion and the differential calculus, in the Principia).

The interval was full of drama, in comparison with which arguments among contemporary cosmologists pale into politeness. There was, for example, the burning of Bruno ${ }^{2}$ at the stake in 1600 in conformity with the Inquisition's routine injunction that he should "be dealt with as mercifully as possible and without the shedding of blood" and Galileo's forced abjuration of the heliocentric hypothesis in 1633 followed by his house imprisonment until his death in 1642 .

Then came Newton's Principia, with a theory of gravity that, for the first time, made possible a rational cosmology, not to mention the application of newtonian mechanics to the calculation of all physical phenomena, celestial mechanics included. There were also, of course, Newton's quarrels with Hooke (over priority for the theory of gravitation) and with Leibniz (over priority for the calculus).

It is notable that there was nothing in the seventeenth century to suggest that the Universe is other than static. The fixed stars were fixed. But by the end of the eighteenth century, William Herschel was able to measure the proper motion and the parallax of nearby stars, showing that

\section{IMAGE UNAVAILABLE FOR COPYRIGHT REASONS}

\section{Telescope of Galileo}

the Milky Way has a motion of its own, with individual objects continuing in what Newton had called their state of "rest or motion".

It is surprising, but true, that when William Herschel managed to resolve into stars some of the 5,000 nebulae he had catalogued by 1820 , nobody took much notice for the best part of a century. The true scale of the Universe was not appreciated until 1930 or thereabouts, when the 100 -inch reflector at Mount Wilson had shown that the Milky Way, "our Galaxy", is mirrored in uncounted others, perhaps 100 million of them, sometimes drawn together into clusters.

The first observations to show that the Universe is not static came earlier, during the First World War, when V.M. Slipher, F. G. Pease and Harlow Shapley, using the new generation of telescopes in the United States, were able to show spectroscopically that the distant galaxies are predominantly moving away from the Sun (or from our Galaxy). Until that point, there would have been no reason for people to abandon the ancient idea that the Universe is fixed for all time, although people such as Jeans were worrying about the longevity of stellar energy sources.

By good luck, that development virtually coincided with the appearance of Einstein's general theory of relativity and was soon followed by de Sitter's demonstra- tion that one solution of Einstein's equa tions is consistent with a picture of an expanding Universe.

During the 1930 s, the great hunt for signs of life on Mars and other planets, which had occupied some of the best telescope time for half a century, was finally abandoned as people turned to contemplate the vast scale of the Universe revealed by the distance of the galaxies derived from the redshifts measured at Mount Wilson, as well as to astrophysical problems, notably the generation of energy by thermonuclear reactions in stars.

Cosmology came into its own again only after the Second World War, and for three यु reasons: the 200-inch Hale telescope on Mount Palomar was commissioned in 1950, making the megaparsec the cosmologists' awesome standard unit of distance; the development of radioastronomy made a novel class of galaxies accessible; and Bondi, Gold and Hoyle published their contentious theory of the steady-state Universe in which matter is continuously created.

For the past 30 years, Big Bang cosmology has become generally accepted as the true world view. It may well be a correct one. But the episodic character of the historical record must prompt the question whether an explanation of the expanding Universe, itself exclusively the creation of this century, is likely to be complete.

In the long history of cosmology, we forget how much dead-time there has been. Some of the delays are those needed for novel concepts to become generally accessible. Thus the importance of the Principia was not fully appreciated outside Britain until the second edition appeared, in 1713. (The greatest among Newton's contemporaries, Christian Huygens, had already rejected the notion of action at a distance implied by Newton's gravitation.) But then newtonian mechanics had to be made usable by the work of Lagrange, Hamilton, Jacobi and the like. Similarly, it is a phenomenon of recent experience that it has taken the best part of half a century for the general theory of relativity to become a part of physics proper.

On other occasions, cosmology has been kept waiting for the arrival of new techniques. Would it have fallen to Galileo to make the decisive arguments for the heliocentric hypothesis if his predecessors had had access to a telescope with which to see Jupiter's satellites? In our own time, each extension of observation to new regions of the electromagnetic spectrum has coloured (and sometimes changed) our world view. Can we be sure that this process is at an end?

\footnotetext{
1 Pannekoek, A. A History of Astronomy (Allen \& Unwin, London, 1961).

2. Snyder, C. The World Machine (Longman, London, 1907).
} 